\title{
Adolescent Overweight and Obesity: Links to Socioeconomic Status and Fruit and Vegetable Intakes
}

\author{
Jihyun You and Jina Choo* \\ College of Nursing, Korea University, Anam-Dong, Seongbuk-Gu, Seoul 137-705, Korea; jhemin@korea.ac.kr \\ * Correspondence: jinachoo@korea.ac.kr; Tel.: +82-2-3290-4925
}

Academic Editor: Maria M. Morales Suarez-Varela

Received: 5 January 2016; Accepted: 1 March 2016; Published: 9 March 2016

\begin{abstract}
Whether adolescent overweight/obesity is linked to socioeconomic status (SES) and fruit and vegetable $(\mathrm{F} / \mathrm{V})$ intakes has not been confirmed. We aimed to determine whether there is an association between SES and adolescent overweight/obesity and to test the mediating effect of F/V intakes. This cross-sectional study included the data of 63,111 adolescents extracted from the 2013 Korea Youth Risk Behavior Web-based Survey. Overweight/obesity was defined as a body mass index $\geqslant 85$ th percentile, while F/V intakes were categorized as high (recommended levels: $\geqslant 1$ fruit serving and $\geqslant 3$ vegetable servings per day) versus low. Among girls, low SES (beta $=0.50, p<0.001$ ) and $\mathrm{F} / \mathrm{V}$ intakes (beta $=-0.17, p=0.038$ ) were both significantly associated with overweight/obesity; the former association was significantly mediated by F/V intakes (Sobel test: $\mathrm{z}=2.00, p=0.046$ ). Among boys, neither SES nor F/V intakes was significantly associated with overweight/obesity. Adolescent overweight/obesity was significantly linked to low SES and F/V intakes among girls only; low SES indirectly increased the risk of overweight/obesity via low F/V intakes. Therefore, promoting $\mathrm{F} / \mathrm{V}$ intakes for socially disadvantaged girls should be prioritized as a population-based strategy for preventing adolescent overweight/obesity in South Korea.
\end{abstract}

Keywords: social class; fruit and vegetable intake; adolescent; obesity; overweight

\section{Introduction}

Adolescent overweight/obesity has emerged as a major public health concern, as its prevalence has increased remarkably across countries of all economic level [1]. In the United States, the prevalence of adolescent overweight/obesity (body mass index (BMI) $\geqslant 85$ th percentile) increased from $30.4 \%$ to $34.5 \%$ between 2000 and 2012 [2,3]. In South Korea, the prevalence of obesity (BMI $\geqslant 95$ th percentile) increased from $9.2 \%$ to $12.7 \%$ between 1998 and 2013 [4].

Adolescent overweight/obesity increases the risk of adult overweight/obesity [5,6]. Specifically, a previous study reported that adolescent obesity at 10-17 years of age was more likely to be associated with the risk for adulthood obesity than childhood obesity at 1-9 years of age [7]. Moreover, adolescent overweight/obesity is reportedly associated with an increased risk of metabolic syndrome in young adulthood and increased risks of cardiovascular disease and cancer in later adulthood [8-10]. Therefore, identifying determinants associated with adolescent overweight/obesity is necessary for preventing its adverse consequences such as latent obesity and morbidity in adulthood.

Socioeconomic status (SES) may be a determinant of adolescent overweight/obesity [11]. A recent systematic review reported that SES and child-adolescent weight status were inversely associated in rich countries and that its magnitude increased with age [12]. Several previous studies, mostly in Europe and the U.S., also revealed that inverse associations between SES and overweight/obesity significantly differed by gender in childhood and adolescence [13,14], showing a stronger association in girls than in boys [13] or a significant association in girls only [14]. Such an inverse association in girls has been speculated due to unmeasured behavioral factors associated with SES [14]. 
Fruit and vegetable $(\mathrm{F} / \mathrm{V})$ intakes are healthy eating behaviors that may be best fostered in childhood and adolescence based on their proven significant health benefits of preventing diabetes mellitus, cardiovascular disease, and some cancers [15-17]. Reportedly, low F/V intakes are a precursor of overweight/obesity [18], while high F/V intakes have beneficial effects on preventing excessive weight gain in adulthood $[19,20]$. Low F/V intakes are reportedly associated with low SES, particularly shown more prevalent in socially disadvantaged adolescent girls than those boys [21]. However, so far, evidence about the links between F/V intakes and overweight/obesity in adolescence with the concomitant consideration of SES is scarce.

We thus posited that low SES and low F/V intakes would be linked to adolescent overweight/obesity and tested whether low F/V intakes would mediate the association between low SES and overweight/obesity in Korean adolescent boys and girls. Specifically, four hypotheses were proposed for the present study: (1) low SES would be significantly associated with overweight/obesity in adolescent boys and girls; (2) low SES would be significantly associated with low F/V intakes in adolescent boys and girls; (3) low F/V intakes would be significantly associated with overweight/obesity in adolescent boys and girls after adjusting for SES; and (4) low F/V intakes would mediate the association between low SES and overweight/obesity in adolescent boys and girls. To investigate these hypotheses, we used nationally representative data of a Korean adolescent population obtained from the 2013 Korea Youth Risk Behavior Web-based Survey (KYRBWS) [22].

\section{Materials and Methods}

\subsection{Design and Participants}

This cross-sectional, population-based study included the data of 63,111 boys and girls that were extracted from the 2013 KYRBWS [22]. The KYRBWS is officially approved by Statistics Korea (certificate number 11758) and is repeated annually by the Korea Centers for Disease Control and Prevention; Ministry of Health and Welfare; and Ministry of Education, Science, and Technology to investigate health-related behaviors of Korean adolescents aged 12-18 years including smoking, alcohol drinking, physical activity, eating behaviors, and weight status [23]. KYRBWS data are collected using a multistage, stratified, cluster-sampling method that involves stratification, clustering, weight, and finite population correction. The population was stratified according to regional and school-type variables, and the sample was then clustered and selected according to school and class [22]. In the KYRBWS, Korean adolescents in grades 7-12 grades at middle and high schools voluntarily and anonymously complete a web-based self-administered questionnaire. Written informed consent was received from all participants and their parents or legal guardians. The online self-reported survey was performed in school computer rooms after the participants were fully informed about the survey and assigned identification numbers to ensure anonymity. The total number of participants in the 2013 KYRBWS was 72,435 boys and girls. After excluding those with missing data, a total of 63,111 boys and girls were included in the final analysis.

\subsection{Measurements}

For the present study, sociodemographic, behavioral, and anthropometric characteristics were extracted from the 2013 KYRBWS data. Sociodemographic characteristics included age, school type (middle or high), learning achievement (low or high), residential setting (city or rural), living arrangement (with or without family), parents' nationality (Korean or foreign), adolescents' perceived SES (high, mid-high, mid, mid-low, or low), and parents' highest education level (categorical: $\leqslant 9,10-12$, or $\geqslant 13$ years). The parents' highest education level was defined as the highest of either the father's or mother's education levels. We combined and recoded the two indicators of adolescents' perceived SES and parents' highest education levels to create a SES variable of low versus high SES: perceived SES was recoded as high (high, mid-high, mid SES) versus low (mid-low, low SES), and parents' highest education levels were recoded as high $(\geqslant 13$ years, college-educated) versus low $(<13$ years, less 
college-educated). In the present study, low SES was defined as both low perceived SES and low parents' highest education, whereas high SES was defined as any other combination. Previously, the SES indicators of adolescents' perceived SES [24,25] and parents' highest education levels $[12,21,26,27]$ were reported to associate significantly with the health status, health behaviors, and overweight/obesity among adolescents. Moreover, these two SES indicators exhibited a significant correlation with each other (Spearman rho $=0.3, p<0.001$ ). In this regard, a combined SES variable involving these two SES indicators might be more robust than each of the indicators alone with respect to associations with $\mathrm{F} / \mathrm{V}$ intakes and overweight/obesity among adolescents; in fact, the combined SES variable was found to be more robust (data not shown).

The behavioral characteristics used in the present study were F/V intakes, fast-food intakes, regular exercise, and sedentary behavior. F/V intakes were measured using two questions: "How often did you eat fruit (not including fruit juice) during the past 7 days?" and "How often did you eat vegetables (not including kimchi) during the past 7 days?", and recoded as high (recommended level: $\geqslant 1$ fruit serving and $\geqslant 3$ vegetable servings per day) versus low (below the recommended level) according to the criteria of the Dietary Guidelines for Adolescents from the Korea Ministry of Health and Welfare [28-30]. Fast food intakes were measured by asking the participants, "How often did you eat fast foods such as fried chicken, hamburgers, or pizza during the recent 7 days?" and recoded as ever-consuming ( $\geqslant 1$ time during the recent 7 days) versus never-consuming [31].

Regular exercise was measured by asking participants, "How often did you exercise moderately and vigorously during the past 7 days?" and recoded as high (recommended level: moderate physical activity every day and vigorous physical activity $\geqslant 3$ days per week) versus low (below the recommended level) according to the criteria of the 2008 Physical Activity Guidelines for Americans from the Centers for Disease Control and Prevention [32,33]. Sedentary behavior was measured by asking, "How many hours did you sit during the recent weekdays?" and recoded as low (recommended level: $<2 \mathrm{~h}$ per day of sedentary time during the recent weekday) versus high (below the recommended level) [33]. All of the recommended levels were defined according to the criteria of the Korea Ministry of Health and Welfare, U.S. Centers for Disease Control and Prevention, and Canada Center for Disease Control and Prevention [28,31-33].

Anthropometric characteristics were obtained as self-reported body weight $(\mathrm{kg})$ and height $(\mathrm{cm})$. BMI was calculated as the weight in kilograms divided by the square of the height in meters $\left(\mathrm{kg} / \mathrm{m}^{2}\right)$, and categorized as non-overweight/obese (BMI $<85$ th percentile) versus overweight/obese (BMI $\geqslant 85$ th percentile) using sex- and age-specific BMI percentiles based on the Korea Centers for Disease Control and Prevention growth charts [34].

\subsection{Ethical Considerations}

The study was approved by the Institutional Review Board at Korea University (KU-IRB-15-EX-254-A-1) and all procedures were followed in accordance with the ethical standards of this board.

\subsection{Data Analysis}

Participants $(n=63,111$, weighted $\mathrm{n}(\mathrm{wn})=3,272,722)$ were included in the final analysis and analyzed by gender-stratified group using the complex samples procedure. "wn" refers to the population size determined by multiplying the values of weight $(\mathrm{w})$ and sample size (n). All statistical analyses were performed using SPSS 23.0 (SPSS Inc., Chicago, IL, USA).

The participants' general characteristics are expressed as either weighted means (standard errors) or numbers (weighted \%) as appropriate. To test differences between boys and girls in the participants' sociodemographic, behavioral, and anthropometric characteristics, an independent $t$-test or chi-square test was used as appropriate. Logistic regressions were performed to examine crude associations of sociodemographic and behavioral characteristics with overweight/obesity and independent associations of SES and F/V intakes with overweight/obesity after adjusting for 
covariates. We included the following covariates in adjusted regression models based on the results of crude associations with overweight/obesity $(p<0.2)$ : age, school type, residential setting, learning achievement, parents' nationality, fast-food intake, regular exercise, and sedentary behavior.

Finally, to test four hypotheses, including the mediating effect of F/V intakes on the association between SES and adolescent overweight/obesity, three equations were examined via logistic regressions [35]. To test Hypothesis 1 (SES would be significantly associated with overweight/obesity in adolescent boys and girls), overweight/obesity (outcome) was regressed on SES (predictor) in the first equation. To test Hypothesis 2 (SES would be significantly associated with F/V intakes in adolescent boys and girls), F/V intakes (outcome) were regressed on SES (predictor) in the second equation. To test Hypothesis 3 (F/V intakes would be significantly associated with overweight/obesity in adolescent boys and girls after adjustment for SES), overweight/obesity (outcome) was regressed on F/V intakes after adjusting for SES in the third equation. To test Hypothesis 4 (F/V intakes would significantly mediate the association between SES and overweight/obesity in adolescent boys and girls), the Sobel test was used to evaluate the potential mediation effect of F/V intakes on the association between SES and overweight/obesity, with regression coefficients and standard errors obtained from the results of Hypotheses 2 and 3. The Sobel test is used to test the significance of a mediation effect by determining whether the reduction in the effect of the predictor after including the mediator in the model is significant and, therefore, whether the mediation effect is statistically significant [36]. Values of $p<0.05$ were considered statistically significant.

\section{Results}

\subsection{Participants' General Characteristics}

The participants ( $n=63,111$, wn $=3,272,722$ ) had a mean age of 14.8 years (range, $12-18$ years); of them, $49.7 \%$ were girls and $46.5 \%$ were high school students (Table 1). Of the total participants, $10.8 \%$ lived in a rural setting. Girls were significantly less likely to be overweight and obese than boys $(13.4 \%$ of girls versus $15.2 \%$ of boys with a BMI $\geqslant 85$ th percentile, $p<0.001)$. Girls were significantly more likely to have low F/V intakes $(p=0.004)$ and low regular exercise $(p<0.001)$, and high sedentary behavior than boys $(p<0.001)$. Moreover, girls were more likely than boys to be of low SES $(p=0.026)$.

Table 1. Participants' general characteristics $(n=63,111)$, wn $=3,272,722$.

\begin{tabular}{|c|c|c|c|c|c|c|c|c|}
\hline & \multicolumn{6}{|c|}{ n (Weighted \%), Mean (SE) } & \multirow{3}{*}{$t / x^{2}$} & \multirow{3}{*}{$p$} \\
\hline & \multicolumn{2}{|c|}{ All } & \multicolumn{2}{|c|}{ Girls } & \multicolumn{2}{|c|}{ Boys } & & \\
\hline & & & \multicolumn{2}{|c|}{$(n=31,343)$} & \multicolumn{2}{|c|}{$(n=31,768)$} & & \\
\hline \multicolumn{9}{|c|}{ Sociodemographic Characteristics } \\
\hline Age & 14.8 & $(0.05)$ & 14.9 & $(0.06)$ & 14.7 & $(0.07)$ & 1.54 & $<0.001$ \\
\hline School type & & & & & & & 7.24 & 0.518 \\
\hline Middle & 31,894 & $(53.5)$ & 16,102 & $(54.0)$ & 15,792 & (52.9) & & \\
\hline High & 31,217 & $(46.5)$ & 15,241 & $(46.0)$ & 15,976 & $(47.1)$ & & \\
\hline Learning achievement & & & & & & & 151.85 & $<0.001$ \\
\hline High & 7057 & (11.5) & 3086 & (9.9) & 3971 & $(13.0)$ & & \\
\hline Low & 56,054 & $(88.5)$ & 28,257 & $(90.1)$ & 27,797 & $(87.0)$ & & \\
\hline Residental setting & & & & & & & 74.67 & 0.166 \\
\hline City & 55,717 & $(89.2)$ & 27,925 & $(90.3)$ & 27,792 & $(88.2)$ & & \\
\hline Rural & 7394 & $(10.8)$ & 3418 & $(9.7)$ & 3976 & $(11.8)$ & & \\
\hline Living arrangement & & & & & & & 30.47 & 0.023 \\
\hline With family & 60,607 & $(96.3)$ & 30,201 & $(96.7)$ & 30,406 & $(95.9)$ & & \\
\hline Without family & 2504 & $(3.7)$ & 1142 & $(3.3)$ & 1362 & $(4.1)$ & & \\
\hline
\end{tabular}


Table 1. Cont.

\begin{tabular}{|c|c|c|c|c|c|c|c|c|}
\hline & \multicolumn{6}{|c|}{ n (Weighted \%), Mean (SE) } & \multirow{3}{*}{$t / \chi^{2}$} & \multirow{3}{*}{$p$} \\
\hline & \multicolumn{2}{|c|}{ All } & \multicolumn{2}{|c|}{ Girls } & \multicolumn{2}{|c|}{ Boys } & & \\
\hline & & & \multicolumn{2}{|c|}{$(n=31,343)$} & \multicolumn{2}{|c|}{$(n=31,768)$} & & \\
\hline & & & & & & & & \\
\hline Korean & 62,460 & $(99.0)$ & 31,010 & $(99.0)$ & 31,450 & $(99.0)$ & & \\
\hline Foreign & 651 & $(1.0)$ & 333 & $(1.0)$ & 318 & $(1.0)$ & & \\
\hline SES & & & & & & & 16.90 & 0.026 \\
\hline High & 55,685 & $(88.6)$ & 27,520 & $(88.1)$ & 28,165 & $(89.1)$ & & \\
\hline Low & 7426 & (11.4) & 3826 & $(11.9)$ & 3603 & $(10.9)$ & & \\
\hline \multicolumn{9}{|l|}{ Behavioral characteristics } \\
\hline $\mathrm{F} / \mathrm{V}$ intakes (per day) ${ }^{1}$ & & & & & & & 11.61 & 0.004 \\
\hline Low & 59,695 & $(94.5)$ & 29,728 & $(94.8)$ & 29,967 & $(94.2)$ & & \\
\hline High & 3416 & $(5.5)$ & 1615 & $(5.2)$ & 1801 & $(5.8)$ & & \\
\hline Fast-food intakes & & & & & & & 4.09 & 0.146 \\
\hline Ever-consuming & 43,530 & $(68.9)$ & 21,484 & $(68.5)$ & 22,046 & $(69.2)$ & & \\
\hline Never-consuming & 19,581 & $(31.1)$ & 9859 & $(31.5)$ & 9722 & (30.8) & & \\
\hline Regular exercise $^{2}$ & & & & & & & 1095.37 & $<0.001$ \\
\hline Low & 60,212 & (95.3) & 30,763 & $(98.1)$ & 29,449 & $(92.5)$ & & \\
\hline High & 2899 & $(4.7)$ & 580 & $(1.9)$ & 2319 & $(7.5)$ & & \\
\hline Sedentary behavior ${ }^{3}$ & & & & & & & 250.57 & $<0.001$ \\
\hline High & 38,207 & $(60.8)$ & 19,982 & $(63.8)$ & 18,225 & $(57.7)$ & & \\
\hline Low & 24,904 & $(39.2)$ & 11,3611 & $(36.2)$ & 13,543 & $(42.3)$ & & \\
\hline \multicolumn{9}{|l|}{ Anthropometric characteristics } \\
\hline BMI & & & & & & & 43.52 & $<0.001$ \\
\hline Non-overweight/obese & 54,076 & $(85.7)$ & 27,145 & $(86.6)$ & 26,931 & $(84.8)$ & & \\
\hline Overweight/obese & 9035 & $(14.3)$ & 4198 & $(13.4)$ & 4837 & $(15.2)$ & & \\
\hline
\end{tabular}

\subsection{Crude Associations of Sociodemographic and Behavioral Characteristics with Overweight/Obesity}

Girls who were younger $(p<0.001)$, enrolled in high schools $(p=0.045)$, reported themselves as learning less-achieved $(p<0.001)$, had low F/V intakes $(p=0.026)$, and never-consumed fast-foods in the recent 7 days $(p<0.001)$ were significantly more likely than their counterparts to be overweight or obese (Table 2). Boys who reported themselves as learning less-achieved $(p<0.001)$, who never-consumed fast-food intakes $(p<0.001)$ during the recent 7 days, and had low regular exercise during the recent 7 days $(p<0.001)$ were significantly more likely than their counterparts to be overweight or obese.

Table 2. Crude associations of sociodemographic and behavioral characteristics with overweight/obesity among adolescents $(n=63,111)$, wn $=3,272,722$.

\begin{tabular}{lccccccc}
\hline \multirow{2}{*}{ Characteristics } & \multicolumn{3}{c}{ Girls $(\boldsymbol{n = 3 1 , 3 4 3 )}$} & \multicolumn{3}{c}{ Boys $(\boldsymbol{n = 3 1 , 7 6 8 )}$} \\
\cline { 2 - 7 } & OR & $\mathbf{( C I )}$ & $\boldsymbol{p}$ & OR & $\mathbf{( C I )}$ & $\boldsymbol{p}$ \\
\hline Sociodemographic Characteristics & & & & & & \\
$\quad$ Age (ref: younger) & 0.84 & $(0.78-0.91)$ & $<0.001$ & 1.07 & $(1.00-1.15)$ & 0.065 \\
School type (ref: middle schools) & 1.08 & $(1.00-1.17)$ & 0.045 & 1.06 & $(0.98-1.14)$ & 0.158 \\
Residential setting (ref: city) & 1.10 & $(1.00-1.22)$ & 0.066 & 0.95 & $(0.83-1.09)$ & 0.467 \\
Living arrangement (ref: with family) & 0.98 & $(0.84-1.15)$ & 0.810 & 0.91 & $(0.77-1.08)$ & 0.282 \\
Learning achievement (ref: high) & 1.38 & $(1.23-1.54)$ & $<0.001$ & 1.22 & $(1.11-1.35)$ & $<0.001$ \\
Parents' nationality (ref: Korean) & 1.11 & $(0.83-1.49)$ & 0.496 & 0.76 & $(0.54-1.07)$ & 0.115 \\
SES (ref: high) & 1.66 & $(1.52-1.80)$ & $<0.001$ & 1.13 & $(1.02-1.25)$ & 0.020 \\
\hline
\end{tabular}


Table 2. Cont.

\begin{tabular}{|c|c|c|c|c|c|c|}
\hline \multirow{2}{*}{ Characteristics } & \multicolumn{3}{|c|}{ Girls $(n=31,343)$} & \multicolumn{3}{|c|}{ Boys $(n=31,768)$} \\
\hline & OR & (CI) & $p$ & OR & (CI) & $p$ \\
\hline \multicolumn{7}{|l|}{ Behavioral Characteristic } \\
\hline $\mathrm{F} / \mathrm{V}$ intakes (ref: low) ${ }^{1}$ & 0.84 & $(0.71-0.98)$ & 0.026 & 0.93 & $(0.81-1.05)$ & 0.233 \\
\hline Fast-food intakes (ref: never-consuming) & 0.76 & $(0.72-0.82)$ & $<0.001$ & 0.86 & $(0.80-0.92)$ & $<0.001$ \\
\hline Regular exercise (ref: low) ${ }^{2}$ & 1.03 & $(0.81-1.31)$ & 0.798 & 0.79 & $(0.69-0.90)$ & $<0.001$ \\
\hline Sedentary behavior (ref: low) ${ }^{3}$ & 1.06 & $(1.00-1.13)$ & 0.071 & 1.04 & $(0.97-1.12)$ & 0.295 \\
\hline
\end{tabular}

\subsection{Testing of Four Hypotheses: Associations among SES, F/V Intakes, and Overweight/Obesity}

Among girls, low SES was significantly and positively associated with overweight/obesity in the first equation (Hypothesis 1$)(B=0.509, p<0.001)$, and significantly and inversely associated with $\mathrm{F} / \mathrm{V}$ intakes in the second equation (Hypothesis 2$)(\mathrm{B}=-0.835, p<0.001)$ (Table 3). In the third equation, $\mathrm{F} / \mathrm{V}$ intakes $(\mathrm{B}=-0.171, p<0.038)$ were significantly and inversely associated with overweight/obesity after adjusting for SES (Hypothesis 3). Moreover, a low SES continued to exert a significant direct influence on overweight/obesity $(B=0.505, p<0.001)$, although the magnitude of this association slightly decreased after adjusting for $\mathrm{F} / \mathrm{V}$ intakes in the third equation (Hypothesis 4 ).

Table 3. Testing for a mediating effect of fruit/vegetable intakes on the association between SES and overweight/obesity among adolescents $(n=63,111)$, wn $=3,272,722$.

\begin{tabular}{|c|c|c|c|c|c|c|}
\hline \multirow{2}{*}{ Variables } & \multicolumn{3}{|c|}{ Girls $^{1}$} & \multicolumn{3}{|c|}{ Boys $^{1}$} \\
\hline & B & SE & $p$ & B & SE & $p$ \\
\hline \multicolumn{7}{|l|}{ First equation } \\
\hline Outcome: overweight/obesity & & & & & & \\
\hline Predictor: low SES & 0.509 & 0.042 & $<0.001$ & 0.093 & 0.052 & 0.076 \\
\hline \multicolumn{7}{|l|}{ Second equation } \\
\hline Outcome: F/V intake & & & & & & \\
\hline Predictor: low SES & -0.835 & 0.113 & $<0.001$ & -0.765 & 0.119 & $<0.001$ \\
\hline \multicolumn{7}{|l|}{ Third equation } \\
\hline Outcome: overweight / obesity & & & & & & \\
\hline Mediator: F/V intake & -0.171 & 0.082 & 0.038 & -0.040 & 0.068 & 0.554 \\
\hline Predictor: low SES & 0.505 & 0.042 & $<0.001$ & 0.092 & 0.053 & 0.082 \\
\hline
\end{tabular}

Hence, F/V intakes had a partial mediating effect on the association between low SES and overweight/obesity among girls (Hypothesis 4) (Sobel test; $\mathrm{z}=2.00, p=0.046$ ) (Figure 1). However, among boys, low SES was not significantly associated with overweight/obesity in the first equation (Hypothesis 1), but was significantly and inversely associated with F/V intakes in the second equation (Hypothesis 2) $(\mathrm{B}=-0.765, p<0.001)$. In the third equation, $\mathrm{F} / \mathrm{V}$ intakes was not significantly associated with overweight/obesity with adjustment for SES (Hypothesis 3). Based on this, we did not conduct a Sobel test for testing Hypothesis 4. 
First equation

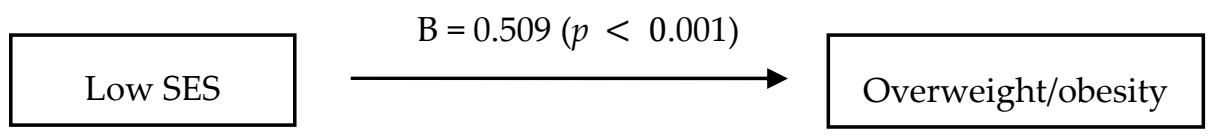

(a)

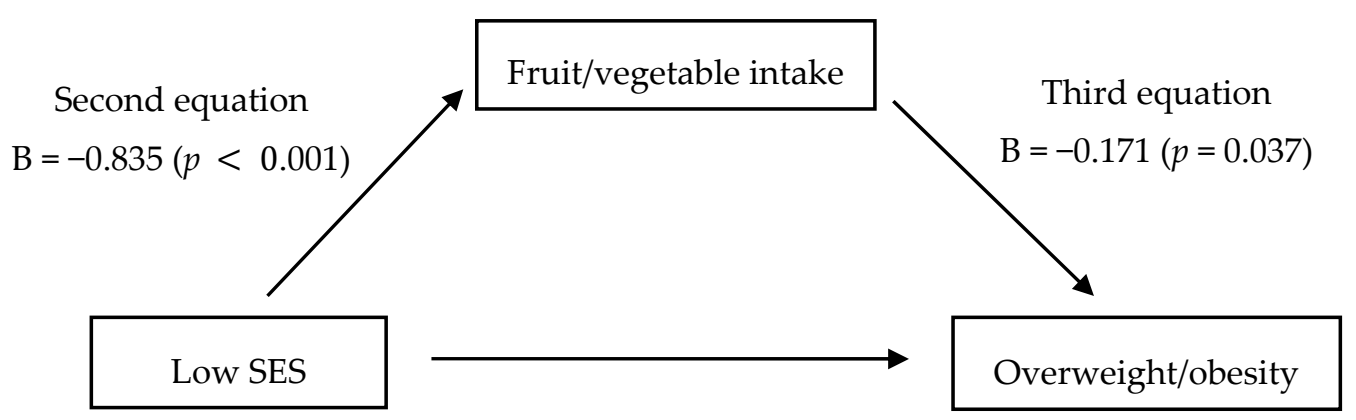

Third equation

$\mathrm{B}=0.505(p<0.001)$

(b)

Sobel test $(\mathrm{z}=\mathbf{2 . 0 0 , p = 0 . 0 4 6 )}$

Figure 1. Adolescent overweight/obesity linked to socioeconomic status and fruit and vegetable intakes in adolescent girls: Testing for a mediating effect of fruit/vegetable intakes on the association between SES and overweight/obesity using a logistic regression analysis (a) Direct pathway from low SES to overweight/obesity; (b) Indirect pathway from low SES to overweight/obesity via fruit/vegetable intakes. B: standardized regression coefficient

\section{Discussion}

In this Korean adolescent population, low SES was significantly associated with overweight/obesity, while low F/V intakes had a significant mediating effect on the association between low SES and overweight/obesity. However, the links between low SES, F/V intakes, and adolescent overweight/obesity differed by gender; among girls, low SES and low F/V intakes were significantly associated with overweight/obesity and low SES was significantly associated with overweight/obesity via low F/V intakes. Among boys, neither low SES nor F/V intakes was significantly associated with overweight/obesity.

We found that low SES was significantly associated with overweight/obesity among adolescent girls but not adolescent boys; in fact, gender significantly modified the association between SES and overweight/obesity in a pooled data of adolescent boys and girls $(p<0.001$; data not shown). This gender-specific association between SES and adolescent overweight/obesity was consistent with the findings of several previous studies $[12,37,38]$, although all studies were not consistent. The Health Behavior in School-aged Children Study, a WHO collaborative cross-national study [39] reported that family affluence, a SES indicator, was inversely associated with overweight/obesity in both boys and girls in approximately half of 39 included countries. However, Singh et al. [13] examined the association of neighborhood SES and built environment with overweight/obesity among 91,642 children aged 10-17 years in the USA and found that the impacts of neighborhood SES and built environment on obesity (not on overweight) were significantly stronger for girls than for boys. Furthermore, Zahnd et al. [14] examined the association between socioeconomic disadvantage and obesity among 
2648 elementary school students in the USA, and found that socioeconomically disadvantaged girls were at a higher risk of obesity than were non-socioeconomically disadvantaged girls; however, such an association was not seen among boys. Such a gender-specific association was attributed to socioeconomically disadvantaged girls having low access to healthy food and physical environments, which, in turn, predisposed them to increased vulnerability to overweight/obesity.

As stated above, our findings demonstrate that the significant association between low SES and adolescent overweight/obesity was partially and significantly mediated by low F/V intakes among adolescent girls. Meanwhile, the non-significant association between low SES and overweight/obesity among adolescent boys in the present study may have been moderated in part by other factors. Specifically, the Korean patriarchal culture [40] may play a role in protecting the effects of low SES on the risk for overweight/obesity, not though low F/V intakes. Traditionally, boys may be prioritized to be served other healthy foods (in contrast, they may also be leniently permitted fast foods that can be purchased by their own allowance) and signed up in activity facilities despite the family being socially disadvantaged, whereas girls may be marginalized instead. Furthermore, son preference value embedded in Asian countries such as Korea and China may explain girls' low access to healthy behaviors. Wang [41] reported that boys in a family were more likely to be highly educated than girls based on the belief that boys provide financial benefits for their families. In this context, we speculate that low educated girls may be less informed of and have fewer healthy lifestyle skills, specifically Asian country context. However, these speculations might not be generalizable to non-Asian country context.

Meanwhile, we also found that low F/V intakes were significantly associated with overweight/obesity among adolescent girls but not boys. Fruit and vegetables are an important source of fiber and contain high amounts of water and low amounts of saturated fat, which are related to reduced energy density, hunger control, and satiety [42]. Heo et al. [20] reported an inverse association between F/V intakes and overweight/obesity among 430,912 adults using the 2007 Behavioral Risk Factor Surveillance Survey in the U.S. Whigham et al. [43] examined the effects of high $\mathrm{F} / \mathrm{V}$ intakes on long-term weight loss using the measure of serum carotenoids, called as a biomarker of $\mathrm{F} / \mathrm{V}$ intakes, and reported that high $\mathrm{F} / \mathrm{V}$ intakes significantly correlated with weight loss for obese adults. Further, this empirical evidence allowed intervention studies to include a high F/V diet as a weight management strategy [42]. However, few studies investigated the association between F/V intakes and overweight/obesity among adolescents. In this reason, we could not discuss a gender-specific association between low $\mathrm{F} / \mathrm{V}$ intakes and overweight/obesity based on the previous literature. However, previous studies elucidated that genetic, behavioral, and parental factors influencing adolescent overweight/obesity differed by gender [44]. In our data, activity behaviors, measured by regular exercise, rather than eating behaviors, measured by $\mathrm{F} / \mathrm{V}$ intakes, were inversely and significantly associated with overweight/obesity among boys $(B=-0.23, p=0.001$ for boys; data not shown), but not among girls. In this context, different behavioral factors may influence the increased risk of overweight/obesity in girls and boys.

To the best of our knowledge, this is the first study to elucidate the mediating effect of F/V intakes on the association between SES and overweight/obesity in a representative adolescent population. Moreover, this study distinguishes itself from previous studies by reporting data after the full adjustment for potential confounding factors (i.e., age, residential setting, learning achievement, fast food intakes, regular exercise, and sedentary behavior) that were, in previous studies, identified in the association between SES and adolescent overweight/obesity $[45,46]$.

The present study had limitations. First, its cross-sectional nature may not guarantee causal inferences about the mediating effect of F/V intakes on the association between SES and overweight/obesity. Therefore, longitudinal research is needed to understand the causal relationships. Second, the self-reported anthropometric data obtained from the 2013 KYRBWS may underestimate (or overestimate) the actual prevalence of overweight/obesity $[47,48]$. Furthermore, F/V intake data may require a concomitant assessment with additional complementary measures of a food frequency 
questionnaire or 24-hour dietary recall. Third, our results may not be generalizable to other ethnic populations or low- and middle-income countries.

\section{Conclusions}

Adolescent overweight/obesity was significantly linked to low SES and F/V intakes. Low SES, specifically via low F/V intakes, may indirectly increase the risk for adolescent overweight/obesity in girls but not boys. Therefore, promoting F/V intakes for socially disadvantaged girls should be prioritized as a population-based strategy for preventing adolescent overweight/obesity in South Korea.

Acknowledgments: This work was supported by the National Research Foundation of Korea (NRF) grant funded by the Korea government (MSIP) (No. NRF-2014R1A2A1A11050974).

Author Contributions: Jina Choo developed the study hypothesis and provided expert consultation on the data analysis and interpretation. Jina Choo and Jihyun You prepared the manuscript draft. Jihyun You was involved in the data analysis. Both authors were involved in the manuscript review, revision, and final approval process.

Conflicts of Interest: The authors declare no conflicts of interest.

\section{References}

1. Kosti, R.I.; Panagiotakos, D.B. The epidemic of obesity in children and adolescents in the world. Cent. Eur. J. Public Health 2006, 14, 151-159. [PubMed]

2. Ogden, C.L.; Carroll, M.D.; Kit, B.K.; Flegal, K.M. Prevalence of childhood and adult obesity in the United States, 2011-2012. JAMA 2014, 311, 806-814. [CrossRef] [PubMed]

3. Ogden, C.L.; Flegal, K.M.; Carroll, M.D.; Johnson, C.L. Prevalence and trends in overweight among US children and adolescents, 1999-2000. JAMA 2002, 288, 1728-1732. [CrossRef] [PubMed]

4. Ministry of Health and Welfare, Korea Center for Disease Control and Prevention. 2012 Korea National Health and Nutrition Examination Survey; Ministry of Health and Welfare, Korea Center for Disease Control and Prevention: Seoul, Korea, 2012.

5. Deshmukh-Taskar, P.; Nicklas, T.A.; Morales, M.; Yang, S.J.; Zakeri, I.; Berenson, G.S. Tracking of overweight status from childhood to young adulthood: The Bogalusa Heart Study. Eur. J. Clin. Nutr. 2006, 60, 48-57. [CrossRef] [PubMed]

6. The, N.S.; Suchindran, C.; North, K.E.; Popkin, B.M.; Gordon-Larsen, P. Association of adolescent obesity with risk of severe obesity in adulthood. JAMA 2010, 304, 2042-2047. [CrossRef] [PubMed]

7. Whitaker, R.C.; Wright, J.A.; Pepe, M.S.; Seidel, K.D.; Dietz, W.H. Predicting obesity in young adulthood from childhood and parental obesity. N. Engl. J. Med. 1997, 337, 869-873. [CrossRef] [PubMed]

8. Biro, F.M.; Wien, M. Childhood obesity and adult morbidities. Am. J. Clin. Nutr. 2010, 91, 1499s-1505s. [CrossRef] [PubMed]

9. Reilly, J.J.; Kelly, J. Long-term impact of overweight and obesity in childhood and adolescence on morbidity and premature mortality in adulthood: Systematic review. Int. J. Obes. (Lond.) 2011, 35, 891-898. [CrossRef] [PubMed]

10. Weiss, R.; Dziura, J.; Burgert, T.S.; Tamborlane, W.V.; Taksali, S.E.; Yeckel, C.W.; Allen, K.; Lopes, M.; Savoye, M.; Morrison, J.; et al. Obesity and the metabolic syndrome in children and adolescents. N. Engl. J. Med. 2004, 350, 2362-2374. [CrossRef] [PubMed]

11. Singh, G.K.; Kogan, M.D.; Van Dyck, P.C.; Siahpush, M. Racial/ethnic, socioeconomic, and behavioral determinants of childhood and adolescent obesity in the United States: Analyzing independent and joint associations. Ann. Epidemiol. 2008, 18, 682-695. [CrossRef] [PubMed]

12. Barriuso, L.; Miqueleiz, E.; Albaladejo, R.; Villanueva, R.; Santos, J.M.; Regidor, E. Socioeconomic position and childhood-adolescent weight status in rich countries: A systematic review, 1990-2013. BMC Pediatr. 2015, 15, 129. [CrossRef] [PubMed]

13. Singh, G.K.; Siahpush, M.; Kogan, M.D. Neighborhood socioeconomic conditions, built environments, and childhood obesity. Health Aff. (Millwood) 2010, 29, 503-512. [CrossRef] [PubMed] 
14. Zahnd, W.E.; Rogers, V.; Smith, T.; Ryherd, S.J.; Botchway, A.; Steward, D.E. Gender-specific relationships between socioeconomic disadvantage and obesity in elementary school students. Prev. Med. (Baltim.) 2015, 81, 138-141. [CrossRef] [PubMed]

15. Bazzano, L.A.; He, J.; Ogden, L.G.; Loria, C.M.; Vupputuri, S.; Myers, L.; Whelton, P.K. Fruit and vegetable intake and risk of cardiovascular disease in US adults: The first National Health and Nutrition Examination Survey Epidemiologic Follow-up Study. Am. J. Clin. Nutr. 2002, 76, 93-99. [PubMed]

16. Llewellyn, A.; Simmonds, M. Childhood obesity as a predictor of morbidity in adulthood: A systematic review and meta-analysis. Obes. Rev. 2016, 17, 56-67. [CrossRef] [PubMed]

17. Van Duyn, M.A.; Pivonka, E. Overview of the health benefits of fruit and vegetable consumption for the dietetics professional: Selected literature. J. Am. Diet. Assoc. 2000, 100, 1511-1521. [CrossRef]

18. Ledoux, T.A.; Hingle, M.D.; Baranowski, T. Relationship of fruit and vegetable intake with adiposity: A systematic review. Obes. Rev. 2011, 12, e143-e150. [CrossRef] [PubMed]

19. Bes-Rastrollo, M.; Martinez-Gonzalez, M.A.; Sanchez-Villegas, A.; de la Fuente Arrillaga, C.; Martinez, J.A. Association of fiber intake and fruit/vegetable consumption with weight gain in a Mediterranean population. Nutrition 2006, 22, 504-511. [CrossRef] [PubMed]

20. Heo, M.; Kim, R.S.; Wylie-Rosett, J.; Allison, D.B.; Heymsfield, S.B.; Faith, M.S. Inverse association between fruit and vegetable intake and BMI even after controlling for demographic, socioeconomic and lifestyle factors. Obes. Facts 2011, 4, 449-455. [CrossRef] [PubMed]

21. Pitel, L.; Madarasova Geckova, A.; Reijneveld, S.A.; van Dijk, J.P. Socioeconomic differences in adolescent health-related behavior differ by gender. J. Epidemiol. 2013, 23, 211-218. [PubMed]

22. Ministry of Education Science and Technology; Ministry of Health and Welfare; Korea Center for Disease Control and Prevention. Korea Youth Risk Behavior Web-based Survey 2013. Available online: http:/ /yhs.cdc.go.kr/new/ (accessed on 19 December 2015).

23. Korea Centers for Disease Control and Prevention. The Statistics of 9th Korea Youth Risk Behavior Web-Based Survey (KYRBWS) in 2013. Available online: http://yhs.cdc.go.kr/ (accessed on 19 December 2015).

24. Goodman, E.; Huang, B.; Schafer-Kalkhoff, T.; Adler, N.E. Perceived socioeconomic status: A new type of identity that influences adolescents' self-rated health. J. Adolesc. Health 2007, 41, 479-487. [CrossRef] [PubMed]

25. Quon, E.C.; McGrath, J.J. Subjective socioeconomic status and adolescent health: A meta-analysis. Health Psychol. 2014, 33, 433-447. [CrossRef] [PubMed]

26. Finger, J.D.; Varnaccia, G.; Tylleskar, T.; Lampert, T.; Mensink, G.B. Dietary behaviour and parental socioeconomic position among adolescents: The German Health Interview and Examination Survey for Children and Adolescents 2003-2006 (KiGGS). BMC Public Health 2015, 15, 498. [CrossRef] [PubMed]

27. Fradkin, C.; Wallander, J.L.; Elliott, M.N.; Tortolero, S.; Cuccaro, P.; Schuster, M.A. Associations between socioeconomic status and obesity in diverse, young adolescents: Variation across race/ethnicity and gender. Health Psychol. 2015, 34, 1-9. [CrossRef] [PubMed]

28. Ministry of Health and Welfare. Health Plan 2020; Ministry of Health and Welfare: Seoul, Korea, 2011.

29. Park, S.; Choi, B.Y.; Wang, Y.; Colantuoni, E.; Gittelsohn, J. School and neighborhood nutrition environment and their association with students' nutrition behaviors and weight status in Seoul, South Korea. J. Adolesc. Health 2013, 53, 655-662. [CrossRef] [PubMed]

30. Bae, S.G.; Kim, J.Y.; Kim, K.Y.; Park, S.W.; Bae, J.; Lee, W.K. Changes in dietary behavior among adolescents and their association with government nutrition policies in Korea, 2005-2009. J. Prev. Med. Public Health 2012, 45, 47-59. [CrossRef] [PubMed]

31. Ministry of Health and Welfare. Dietary Guidelines for Adolescent; Ministry of Health and Welfare: Seoul, Korea, 2009.

32. Centers for Disease Control and Prevention. 2008 Physical Activity Guidelines for Americans; Centers for Disease Control and Prevention: Atlanta, GA, USA, 2008.

33. Tremblay, M.S.; LeBlanc, A.G.; Janssen, I.; Kho, M.E.; Hicks, A.; Murumets, K.; Colley, R.C.; Duggan, M. Canadian sedentary behaviour guidelines for children and youth. Appl. Physiol. Nutr. Metab. 2011, 36, 59-64. [CrossRef] [PubMed]

34. Korea Center for Disease Control and Prevention; The Korean Pediatric Society. 2007 Korean National Growth Charts; Korea Center for Disease Control and Prevention, The Korean Pediatric Society: Seoul, Korea, 2007. 
35. Baron, R.M.; Kenny, D.A. The moderator-mediator variable distinction in social psychological research: conceptual, strategic, and statistical considerations. J. Pers. Soc. Psychol. 1986, 51, 1173-1182. [CrossRef] [PubMed]

36. Sobel, M.E. Asymptotic confidence intervals for indirect effects in structural equation models. Sociol. Methodol. 1982, 13, 290-312. [CrossRef]

37. Frederick, C.B.; Snellman, K.; Putnam, R.D. Increasing socioeconomic disparities in adolescent obesity. Proc. Natl. Acad. Sci. USA 2014, 111, 1338-1342. [CrossRef] [PubMed]

38. Stamatakis, E.; Wardle, J.; Cole, T.J. Childhood obesity and overweight prevalence trends in England: Evidence for growing socioeconomic disparities. Int. J. Obes. (Lond.) 2010, 34, 41-47. [CrossRef] [PubMed]

39. Currie, C. Social Determinants of Health and Well-Being among Young People; World Health Organization Regional Office for Europe: Copenhagen, Denmark, 2012.

40. Soh, C.-H.S. Sexual equality, male superiority, and Korean women in politics: Changing gender relations in a "patriarchal democracy". Sex Roles. 1993, 28, 73-90. [CrossRef]

41. Wang, W. Son preference and educational opportunities of children in China-“I wish you were a boy!". Gend. Issues 2005, 22, 3-30. [CrossRef]

42. Rolls, B.J.; Ello-Martin, J.A.; Tohill, B.C. What can intervention studies tell us about the relationship between fruit and vegetable consumption and weight management? Nutr. Rev. 2004, 62, 1-17. [CrossRef] [PubMed]

43. Whigham, L.D.; Valentine, A.R.; Johnson, L.K.; Zhang, Z.; Atkinson, R.L.; Tanumihardjo, S.A. Increased vegetable and fruit consumption during weight loss effort correlates with increased weight and fat loss. Nutr. Diabetes. 2012, 2, e48. [CrossRef] [PubMed]

44. Sweeting, H.N. Gendered dimensions of obesity in childhood and adolescence. Nutr. J. 2008, 7, 1. [CrossRef] [PubMed]

45. Sallis, J.F.; Glanz, K. The role of built environments in physical activity, eating, and obesity in childhood. Future. Child. 2006, 16, 89-108. [CrossRef] [PubMed]

46. Campbell, M.K. Biological, environmental, and social influences on childhood obesity. Pediatr. Res. 2016. [CrossRef] [PubMed]

47. Bae, J.; Joung, H.; Kim, J.Y.; Kwon, K.N.; Kim, Y.; Park, S.W. Validity of self-reported height, weight, and body mass index of the Korea Youth Risk Behavior Web-based Survey questionnaire. J. Prev. Med. Public. Health 2010, 43, 396-402. [CrossRef] [PubMed]

48. Sherry, B.; Jefferds, M.E.; Grummer-Strawn, L.M. Accuracy of adolescent self-report of height and weight in assessing overweight status: A literature review. Arch. Pediatr. Adolesc. Med. 2007, 161, 1154-1161. [CrossRef] [PubMed]

(C) 2016 by the authors; licensee MDPI, Basel, Switzerland. This article is an open access article distributed under the terms and conditions of the Creative Commons by Attribution (CC-BY) license (http:/ / creativecommons.org/licenses/by/4.0/). 